\title{
On Golden Lorentzian Manifolds Equipped with Generalized Symmetric Metric Connection
}

\author{
Majid Ali Choudhary ${ }^{1, *}$, Khaled Mohamed Khedher ${ }^{2}{ }^{\mathbb{D}}$, Oğuzhan Bahadır ${ }^{3, *}$ and Mohd Danish Siddiqi ${ }^{4}$ \\ 1 Department of Mathematics, School of Sciences, Maulana Azad National Urdu University, \\ Hyderabad 500032, India \\ 2 Department of Civil Engineering, College of Engineering, King Khalid University, Abha 61421, Saudi Arabia; \\ kkhedher@kku.edu.sa \\ 3 Department of Mathematics, Faculty of Arts and Sciences, Kahramanmaras Sutcu Imam University, \\ Kahramanmaras 46050, Turkey \\ 4 Department of Mathematics, College of Science, Jazan University, Jazan 45142, Saudi Arabia; \\ msiddiqi@jazanu.edu.sa \\ * Correspondence: majid_alichoudhary@yahoo.co.in or majid_alichoudhary@manuu.edu.in (M.A.C.); \\ oguzbaha@gmail.com or obahadir@ksu.edu.tr (O.B.)
}

check for updates

Citation: Choudhary, M.A.; Khedher, K.M.; Bahadır, O.; Siddiqi, M.D. On Golden Lorentzian Manifolds Equipped with Generalized Symmetric Metric Connectio. Mathematics 2021, 9, 2430. https: / / doi.org/10.3390/ math9192430

Academic Editor: Gabriel Eduard Vilcu

Received: 16 July 2021

Accepted: 23 August 2021

Published: 30 September 2021

Publisher's Note: MDPI stays neutral with regard to jurisdictional claims in published maps and institutional affiliations.

Copyright: (C) 2021 by the authors Licensee MDPI, Basel, Switzerland. This article is an open access article distributed under the terms and conditions of the Creative Commons Attribution (CC BY) license (https:// creativecommons.org/licenses/by/ $4.0 /)$.

\begin{abstract}
This research deals with the generalized symmetric metric $U$-connection defined on golden Lorentzian manifolds. We also derive sharp geometric inequalities that involve generalized normalized $\delta$-Casorati curvatures for submanifolds of golden Lorentzian manifolds equipped with generalized symmetric metric $U$-connection.
\end{abstract}

Keywords: Riemannian manifolds; golden structure; scalar curvature

\section{Background}

Golden ratios have been investigated by different researchers for many centuries. Taking inspiration from golden mean, the golden structure introduced by [1] as a polynomial structure [2,3] came into picture, and the structure polynomial was written as $\varphi^{2}=\varphi+I$, $\varphi$ being $(1,1)$ tensor field. In 2007, the authors in [4] investigated invariant submanifolds isometrically immersed in golden Riemannian manifolds, highlighting new ideas. In the same ambient manifold [5], work was carried out on an induced structure, producing some new studies. Recently, Bahadir and Sirajuddin et al. [6] studied slant submanifolds in golden Riemannian manifolds, developing different useful results. The study of golden structures was also carried out on semi-Riemannian manifolds by M. Ozkan [7]. One can refer to [8-11], etc., and the references therein for recent developments in golden differential geometry.

Another important development in the submanifold theory was the introduction of $\delta$-invariants [12] (also known as Chen's invariants). With the help of these new Riemannian invariants, B. Y. Chen not only established a relation in the form of an optimal inequality but also defined and studied a new concept known as ideal immersion. This investigation of Chen's invariants have been extensively used by many researchers in different ambient spaces ([13-15], etc.).

It is known that Gauss curvature might vanish for intuitively curved looking surfaces. That is why F. Casorati [16] introduced another notion that is known today as the Casorati curvature. In 2007, Decu et al. [17] studied optimal inequalities and produced the notions of normalized Casorati curvatures (see also [18]). The same authors obtained an extension of the above results and produced the notion of generalized normalized $\delta$-Casorati curvatures [19]. Later on, these research techniques were used for establishing optimal inequalities by many researchers in different ambient spaces ([20-22], etc.).

The present study deals with the generalized symmetric metric $U$-connection on golden Lorentzian manifolds. We also study the lower bounds for submanifolds immersed 
in golden Lorentzian manifolds equipped with generalized symmetric metric $U$-connection. Moreover, submanifolds for which equality holds are also discussed.

\section{Preliminaries}

Consider the Riemannian manifolds $\left(N^{m}, g\right)$ and $\left(M^{n}, g\right)$ such that $N$ isometrically immerses in $M$. Represent the shape operator of $N$ by $S_{\tilde{\xi}}, \xi \in \Gamma\left(T^{\perp} N\right)$. Further, on $M$, assume that $\bar{\nabla}$ stands for the Levi-Civita connection and $\nabla^{\perp}$ for connection in a normal bundle. Furthermore, for all $Y_{1}, Y_{2} \in \Gamma(T N)$ and $\xi \in \Gamma\left(T^{\perp} N\right)$, we write [23]

$$
\begin{gathered}
\bar{\nabla}_{Y_{1}} Y_{2}=\nabla_{Y_{1}} Y_{2}+h\left(Y_{1}, Y_{2}\right), \\
\bar{\nabla}_{Y_{1} \xi}=-S_{\xi} Y_{1}+\nabla_{Y_{1}}^{\perp} \xi,
\end{gathered}
$$

and

$$
g\left(S_{\xi} Y_{1}, Y_{2}\right)=g\left(h\left(Y_{1}, Y_{2}\right), \xi\right)
$$

One can also recall the Gauss equation as [23]

$$
\begin{aligned}
\bar{R}\left(Y_{1}, Y_{2}, Y_{3}, Y_{4}\right)= & R\left(Y_{1}, Y_{2}, Y_{3}, Y_{4}\right)-g\left(h\left(Y_{1}, Y_{4}\right), h\left(Y_{2}, Y_{3}\right)\right) \\
& +g\left(h\left(Y_{1}, Y_{3}\right), h\left(Y_{2}, Y_{4}\right)\right), \quad \forall Y_{i} \in \Gamma(T N), i=1,2,3,4 .
\end{aligned}
$$

Let us fix $\left\{E_{1}, \ldots, E_{m}, E_{m+1}, \ldots, E_{n}\right\}$ for a local orthonormal frame in $M^{n}$. Define the scalar curvature $\tau$ as follows

$$
\tau=\sum_{1 \leq i<j \leq m} R\left(E_{i}, E_{j}, E_{j}, E_{i}\right) .
$$

Set

$$
\rho=\frac{2}{\left(m^{2}-m\right)} \tau
$$

as the normalized scalar curvature and

$$
\mathcal{H}=\sum_{i=1}^{m} \frac{1}{m} h\left(E_{i}, E_{i}\right)
$$

as the mean curvature. For our convenience, put $h_{i j}^{r}=g\left(h\left(E_{i}, E_{j}\right), E_{r}\right)$. In this way, $\forall r \in\{m+1, \ldots, n\}$, we can write

$$
\|\mathcal{H}\|^{2}=\frac{1}{m^{2}} \sum_{r=m+1}^{n}\left(\sum_{i=1}^{m} h_{i i}^{r}\right)^{2}
$$

and

$$
\|h\|^{2}=\sum_{r=m+1}^{n} \sum_{i, j=1}^{m}\left(h_{i j}^{r}\right)^{2} .
$$

One recalls the Casorati curvature for the submanifold $N$ as

$$
\mathcal{C}=\frac{1}{n}\|h\|^{2} .
$$

Let us represent any linear subspace of $T N$ with the help of $\mathcal{A}$ and fix its dimension equal to $t$ such that $t \geq 2$, spanned by $\left\{E_{1}, \ldots, E_{t}\right\}$. Then, for a $t$-plane section $\mathcal{A}$, one can define the scalar curvature by

$$
\tau(\mathcal{A})=\sum_{1 \leq i<j \leq t} R\left(E_{i}, E_{j}, E_{j}, E_{i}\right),
$$




$$
\mathcal{C}(\mathcal{A})=\frac{1}{t} \sum_{r=m+1}^{n} \sum_{i, j=1}^{t}\left(h_{i j}^{r}\right)^{2} .
$$

Let $\mathcal{L}$ be a hyperplane of $T_{p} N$. Then, Decu et al. [17] introduced the normalized $\delta$-Casorati curvatures as

$$
\begin{gathered}
{\left[\delta_{\mathcal{C}}(m-1)\right]_{p}=\frac{1}{2} \mathcal{C}_{p}+\frac{m+1}{2 m(m-1)} \inf \{\mathcal{C}(\mathcal{L})\},} \\
{\left[\widehat{\delta}_{\mathcal{C}}(m-1)\right]_{p}=2 \mathcal{C}_{p}-\frac{2 m-1}{2 m} \sup \{\mathcal{C}(\mathcal{L})\} .}
\end{gathered}
$$

If $r \neq m(m-1)$ is a positive real number, then according to [19] the generalized normalized $\delta$-Casorati curvatures

$$
\begin{aligned}
{\left[\delta_{\mathcal{C}}(r ; m-1)\right]_{p} } & =r \mathcal{C}_{p} \\
& +\frac{\left(m^{2}-m-r\right)(m+r)(m-1)}{r m} \inf \{\mathcal{C}(\mathcal{L})\},
\end{aligned}
$$

if $\left(m^{2}-m\right)>r>0$ and

$$
\begin{aligned}
{\left[\widehat{\delta}_{\mathcal{C}}(r ; m-1)\right]_{p} } & =r \mathcal{C}_{p} \\
& +\frac{\left(m^{2}-m-r\right)(m+r)(m-1)}{r m} \sup \{\mathcal{C}(\mathcal{L})\}
\end{aligned}
$$

provided $r>\left(m^{2}-m\right)$.

We refer to [24] for further definitions and formulas.

\subsection{Golden Riemannian Manifolds}

We identify a Riemannian manifold with the help of $\left(M^{n}, g\right)$ and of a $(1,1)$-tensor field with G satisfying $[4,6,10,11]$.

$$
K(Y)=Y^{n}+a_{n} Y^{n-1}+\ldots+a_{2} Y+a_{1} I=0,
$$

where I stands for identity transformation, and at $p \in M($ for $Y=\mathrm{G}), \mathrm{G}^{n-1}(p), \mathrm{G}^{n-2}(p), \ldots, \mathrm{G}(p), I$ are linearly independent. Then $K(Y)$ represents structure polynomial.

Further, $M$ is equipped with a golden structure if [6].

$$
\varphi^{2}=\varphi+I
$$

In the above equation, I denotes the identity transformation, and $\varphi$ stands for $(1,1)$ tensor field. When $(M, g)$ is equipped with the golden structure $\varphi, M$ becomes golden Riemannian manifold if the following relations holds good for $\varphi$-compatible metric

$$
g\left(\varphi Y_{1}, Y_{2}\right)=g\left(Y_{1}, \varphi Y_{2}\right), \quad \forall Y_{1}, Y_{2} \in \Gamma(T M),
$$

and

$$
g\left(\varphi Y_{1}, \varphi Y_{2}\right)=g\left(\varphi^{2} Y_{1}, Y_{2}\right)=g\left(\varphi Y_{1}, Y_{2}\right)+g\left(Y_{1}, Y_{2}\right)
$$


The Riemannian curvature tensor $R$ of the locally golden space form $\overline{\mathcal{M}}=\mathcal{M}_{1}\left(c_{1}\right) \times$ $\mathcal{M}_{2}\left(c_{2}\right)$ is written according to [11] as

$$
\begin{aligned}
& R(X, Y) Z=\frac{(\mp \sqrt{5}+3) c_{1}+( \pm \sqrt{5}+3) c_{2}}{10}[g(Y, Z) X-g(X, Z) Y] \\
& +\frac{( \pm \sqrt{5}-1) c_{1}+(\mp \sqrt{5}-1) c_{2}}{10}[g(\varphi Y, Z) X-g(\varphi X, Z) Y \\
& +g(Y, Z) \varphi X-g(X, Z) \varphi Y] \\
& +\frac{c_{1}+c_{2}}{5}[g(\varphi Y, Z) \varphi X-g(\varphi X, Z) \varphi Y] .
\end{aligned}
$$

We define the golden Lorentzian manifold.

Definition 1. Let us consider a semi-Riemannian manifold $\left(M^{n}, g\right)$, where $g$ has the signature $(-,+,+, \ldots,+(n-1$ times $))$. Then $M$ stands for golden Lorentzian manifold if it is endowed with a golden structure $\varphi$ and $g$ is $\varphi$-compatible.

Example 1. Let $\mathbb{R}_{1}^{3}$ represent the semi-Euclidean space and consider the signature of $g$ as $(-,+,+)$. If $\Phi$ stands for a $(1,1)$ tensor field, then it is easy to show that if

$$
\Phi\left(s_{1}, s_{2}, s_{3}\right)=\frac{1}{2}\left(s_{1}+\sqrt{5} s_{2}, s_{2}+\sqrt{5} s_{1}, 2 \psi s_{3}\right),
$$

for any vector field $\left(s_{1}, s_{2}, s_{3}\right) \in \mathbb{R}_{1}^{3}$, where $\psi=\frac{1+\sqrt{5}}{2}$ is the golden mean, then

$$
\Phi^{2}=\phi+I,
$$

and hence, $\Phi$ is a golden structure on $\mathbb{R}_{1}^{3}$. Moreover, $g$ may also be verified to be $\Phi$-compatible. Thus, $\left(\mathbb{R}_{1}^{3}, g, \Phi\right)$ becomes a golden Lorentzian manifold.

Example 2. For any semi-Euclidean space $R_{1}^{5}$ and $(1,1)$ tensor field $\Phi$ defined on $R_{1}^{5}$ as

$$
\Phi\left(s_{1}, s_{2}, s_{3}, s_{4}, s_{5}\right)=\left(\psi s_{1},(1-\psi) s_{2}, \psi s_{3},(1-\psi) s_{4}, \psi s_{5}\right),
$$

where $\psi$ is golden mean. We can see that $\Phi^{2}=\Phi+I$. Moreover, $g$ is $\Phi$-compatible, where $g$ is used for the metric tensor with signature $(-,+,+,+,+)$ on $R_{1}^{5}$. Hence, $\left(R_{1}^{5}, g, \Phi\right)$ represents a golden Lorentzian manifold.

According to the study in [8], we have

Theorem 1. On any golden Lorentzian manifold $(M, g, \varphi)$, the golden structure $\varphi$ is integrable if and only if

$$
\nabla \varphi=0 .
$$

where $\nabla$ denotes the Levi-Civita connection associated with $g$.

The structure $\varphi$ has been considered an integrable golden structure in this work.

\subsection{Generalized Symmetric Metric (g.s.m.) Connection in a Golden Lorentzian Manifold}

Let $(M, g, \varphi)$ denote a golden Lorentzian manifold. We write its associated torsion tensor $\bar{T}$ by

$$
\bar{T}\left(Y_{1}, Y_{2}\right)=-\alpha\left\{u\left(Y_{1}\right) Y_{2}-u\left(Y_{2}\right) Y_{1}\right\}-\beta\left\{u\left(Y_{1}\right) \varphi Y_{2}-u\left(Y_{2}\right) \varphi Y_{1}\right\},
$$

where $Y_{i}$ belong to $\Gamma(T M)$ for $i=1,2$, and $\alpha, \beta$ represent smooth functions on the manifold $M$. In the present scenario, $\bar{\nabla}$ is known as generalized symmetric connection. One can also write the relation

$$
u\left(Y_{1}\right)=g\left(U, Y_{1}\right)
$$


where $U$ is a unitary (spacelike or timelike) vector field on $M$, and $u$ stands for a 1-form. In addition to this, if

$$
\bar{\nabla} g=0,
$$

then $\bar{\nabla}$ represents a generalized metric connection. Otherwise, it is said to be a non-metric connection. Considering $\nabla$ as a Levi-Civita connection, one notices that

$$
\bar{\nabla}_{Y_{1}} Y_{2}=\nabla_{Y_{1}} Y_{2}+H\left(Y_{1}, Y_{2}\right), \forall Y_{1}, Y_{2} \in \Gamma(T M)
$$

Suppose that $H$ presents any $(1,2)$ tensor and let $\bar{T}$ be a torsion tensor of the generalized symmetric metric (g.s.m.) connection $\bar{\nabla}$. Then one obtains

$$
H\left(Y_{1}, Y_{2}\right)=\frac{1}{2}\left[\bar{T}\left(Y_{1}, Y_{2}\right)+T^{\prime}\left(Y_{2}, Y_{1}\right)+T^{\prime}\left(Y_{1}, Y_{2}\right)\right], \forall Y_{1}, Y_{2} \in \Gamma(T M)
$$

and

$$
g\left(\bar{T}\left(Y_{3}, Y_{1}\right), Y_{2}\right)=g\left(T^{\prime}\left(Y_{1}, Y_{2}\right), Y_{3}\right), \forall Y_{1}, Y_{2}, Y_{3} \in \Gamma(T M)
$$

This way Equations (3) and (6) produce the following

$$
T^{\prime}\left(Y_{1}, Y_{2}\right)=\beta\left\{u\left(Y_{1}\right) \phi Y_{2}-g\left(\phi Y_{1}, Y_{2}\right) U\right\}-\alpha\left\{g\left(Y_{1}, Y_{2}\right) U-u\left(Y_{1}\right) Y_{2}\right\}, \forall Y_{1}, Y_{2} \in \Gamma(T M) .
$$

Using Equations (3), (5) and (7), we obtain

$$
H\left(Y_{1}, Y_{2}\right)=\beta\left\{u\left(Y_{2}\right) \phi Y_{1}-g\left(\phi Y_{1}, Y_{2}\right) U\right\}-\alpha\left\{g\left(Y_{1}, Y_{2}\right) U-u\left(Y_{2}\right) Y_{1}\right\}, \forall Y_{1}, Y_{2} \in \Gamma(T M) .
$$

So, one may write

Theorem 2. If $(M, g, \varphi)$ is a golden Lorentzian manifold and $\bar{\nabla}$ denotes the g.s.m. connection of $(\alpha, \beta)$-type, then we have

$$
\bar{\nabla}_{Y_{1}} Y_{2}=\nabla_{Y_{1}} Y_{2}+\alpha \kappa\left(Y_{1}, Y_{2}\right)+\beta \kappa\left(\phi Y_{1}, Y_{2}\right), \forall Y_{1}, Y_{2} \in \Gamma(T M)
$$

where $\kappa\left(Y_{1}, Y_{2}\right)=-g\left(Y_{1}, Y_{2}\right) U+u\left(Y_{2}\right) Y_{1}$.

Corollary 1. (i) In view of Equation (8), when

- $(\alpha, \beta)=(1,0)$, one obtains a semi-symmetric metric connection;

- $(\alpha, \beta)=(0,1)$, one obtains a quarter symmetric metric connection.

(ii) Again using Equation (8), the g.s.m. connection will be

- $\quad \alpha$ semi symmetric metric connection with $\beta=0$;

- $\quad \beta$ quarter symmetric metric connection provided $\alpha=0$.

Definition 2. Let $(M, g, \varphi)$ be golden Lorentzian manifold equipped with a golden structure $\varphi$ and $\bar{\nabla}$ be the g.s.m. connection of $(\alpha, \beta)$-type. If the associated vector field $U$ is parallel, then $\bar{\nabla}$ is called the generalized symmetric metric U-connection on $M$.

Now, when the associated vector field $U$ in Equation (3) satisfies the following relation

$$
g(U, U)=u(U)=\varepsilon,
$$

where $\varepsilon= \pm 1$. Taking into use Theorem 2 and the fact that $U$ is parallel w.r.t. $\bar{\nabla}$, we obtain

$$
\bar{\nabla}_{Y_{1}} U=0 \Leftrightarrow \nabla Y_{1} U=-\alpha \kappa\left(Y_{1}, U\right)-\beta \kappa\left(\varphi Y_{1}, U\right), \forall Y_{1} \in \Gamma(T M) .
$$


Using Equations (8)-(10), we have

$$
\left(\bar{\nabla}_{Y_{1}} u\right) Y_{2}=\left(\nabla_{Y_{1}} u\right) Y_{2}-\alpha u\left(\kappa\left(Y_{1}, Y_{2}\right)\right)-\beta u\left(\kappa\left(\varphi Y_{1}, Y_{2}\right)\right), \forall Y_{1}, Y_{2} \in \Gamma(T M) .
$$

Furthermore, $\left(\bar{\nabla}_{Y_{1}} u\right) Y_{2}=0$, and thus,

$$
\left(\nabla_{Y_{1}} u\right) Y_{2}=\alpha u\left(\kappa\left(Y_{1}, Y_{2}\right)\right)+\beta u\left(\kappa\left(\varphi Y_{1}, Y_{2}\right)\right), \forall Y_{1}, Y_{2} \in \Gamma(T M)
$$

Proposition 1. For any golden Lorentzian manifold $\left(M^{n}, g, \varphi\right)$ with generalized symmetric metric U-connection $\bar{\nabla}$, we have

$$
\begin{aligned}
R\left(Y_{1}, Y_{2}\right) U= & \beta^{2}\left[u\left(\varphi Y_{2}\right) \varphi Y_{1}-u\left(\varphi Y_{1}\right) \varphi Y_{2}\right] \\
+ & \alpha \beta\left[-u\left(Y_{1}\right) \varphi Y_{2}+u\left(Y_{2}\right) \varphi Y_{1}+u\left(\varphi Y_{2}\right) Y_{1}-u\left(\varphi Y_{1}\right) Y_{2}\right] \\
-\quad & \alpha^{2}\left[u\left(Y_{1}\right) Y_{2}-u\left(Y_{2}\right) Y_{1}\right], \\
R\left(U, Y_{1}\right) Y_{2}= & -\alpha^{2} \kappa\left(Y_{1}, Y_{2}\right) \beta^{2}\left[u\left(\varphi Y_{2}\right) \varphi Y_{1}+u\left(\varphi Y_{1}\right) \varphi Y_{2}\right] \\
+ & \alpha \beta\left[-u\left(\varphi Y_{2}\right) Y_{1}-\kappa\left(\varphi Y_{1}, Y_{2}\right)+g\left(Y_{1}, Y_{2}\right) \varphi U\right], \\
u\left(R\left(Y_{1}, Y_{2}\right) Y_{3}\right)= & -\beta^{2}\left[u\left(\varphi Y_{2}\right) g\left(\varphi Y_{1}, Y_{3}\right)-u\left(\varphi Y_{1}\right) g\left(\varphi Y_{2}, Y_{3}\right)\right] \\
& -\alpha \beta\left[u\left(Y_{2}\right) g\left(\varphi Y_{1}, Y_{3}\right)-u\left(Y_{1}\right) g\left(\varphi Y_{2}, Y_{3}\right)\right. \\
& \left.+u\left(\varphi Y_{2}\right) g\left(Y_{1}, Y_{3}\right)-u\left(\varphi Y_{1}\right) g\left(Y_{2}, Y_{3}\right)\right] \\
& +\alpha^{2}\left[u\left(Y_{1}\right) g\left(Y_{2}, Y_{3}\right)-u\left(Y_{2}\right) g\left(Y_{1}, Y_{3}\right)\right],
\end{aligned}
$$

where $R$ is the Curvature tensor.

Proof. Using Equations (9)-(11), we have Equation (12). With the aid of Equation (12), we can easily find the Equations (13) and (14).

Let $M$ be a golden Lorentzian manifold with generalized symmetric metric $U$-connection $\bar{\nabla}$. Let $\bar{R}$ denote the curvature tensor w.r.t. $\bar{\nabla}$. Then, for all $Y_{1}, Y_{2}, Y_{3} \in \Gamma(T M)$, we write

$$
\bar{R}\left(Y_{1}, Y_{2}\right) Y_{3}=\bar{\nabla}_{Y_{1}} \bar{\nabla}_{Y_{2}} Y_{3}-\bar{\nabla}_{Y_{2}} \bar{\nabla}_{Y_{1}} Y_{3}-\bar{\nabla}_{\left[Y_{1}, Y_{2}\right]} Y_{3} \text {. }
$$

Due to Equations (8) and (15), we reach

$$
\begin{aligned}
\bar{R}\left(Y_{1}, Y_{2}\right) Y_{3}= & R\left(Y_{1}, Y_{2}\right) Y_{3}+\alpha^{2}\left\{g\left(Y_{1}, Y_{3}\right) Y_{2}-g\left(Y_{2}, Y_{3}\right) Y_{1}\right\} \\
& +\beta^{2}\left\{g\left(\varphi Y_{1}, Y_{3}\right) \varphi Y_{2}-g\left(\varphi Y_{2}, Y_{3}\right) \varphi Y_{1}\right\} \\
& +\alpha \beta\left\{g\left(\varphi Y_{1}, Y_{3}\right) Y_{2}-g\left(\varphi Y_{2}, Y_{3}\right) Y_{1}\right. \\
& \left.+g\left(Y_{1}, Y_{3}\right) \varphi Y_{2}-g\left(Y_{2}, Y_{3}\right) \varphi Y_{1}\right\} .
\end{aligned}
$$

Considering an orthonormal frame field on $M$ and contracting over $Y$, one can write the Ricci tensor identified by $\bar{S}$ as

$$
\begin{aligned}
\bar{S}\left(Y_{2}, Y_{3}\right)= & S\left(Y_{2}, Y_{3}\right)+\alpha^{2}(1-n \varepsilon) g\left(Y_{2}, Y_{3}\right) \\
& +\beta^{2}\left\{g\left(\varphi Y_{2}, \varphi Y_{3}\right)-g\left(\varphi Y_{2}, Y_{3}\right) \text { trace } \varphi\right\} \\
& +\alpha \beta\left\{(2-n \varepsilon) g\left(\varphi Y_{2}, Y_{3}\right)-g\left(Y_{2}, Y_{3}\right) \text { trace } \varphi\right\},
\end{aligned}
$$

and the Ricci operator by

$$
\begin{aligned}
\bar{Q} Y_{2}= & Q Y_{2}+\alpha^{2}(1-n \varepsilon) Y_{2}+\beta^{2}\left\{\varphi Y_{2}+Y-\varphi Y_{2} \operatorname{trace} \varphi\right\} \\
& +\alpha \beta\left\{(2-n \varepsilon) \varphi Y_{2}-Y_{2} \operatorname{trace} \varphi\right\}
\end{aligned}
$$


Let us identify with $R$ the curvature tensor and denote by $S$ the Ricci tensor w.r.t. Levi-Civita connection. Then, the contraction of Equation (16) along $Y$ helps us to write the scalar curvature with respect to $\bar{\nabla}$, as follows

$$
\begin{aligned}
\bar{\tau}= & \tau+\alpha^{2}(\varepsilon-n) n+\beta^{2}\left\{\operatorname{trace} \varphi+n \varepsilon-(\operatorname{trace} \varphi)^{2}\right\} \\
& +\alpha \beta(2-2 n \varepsilon) \text { trace } \varphi .
\end{aligned}
$$

in the above relations, $Q$ represents Ricci operator, and $\tau$ stands for scalar curvature associated to Levi-Civita connection.

Theorem 3. For any golden Lorentzian manifold equipped with generalized symmetric metric U-connection, we have the following characterizations in Table 1.

Table 1. Characterizations of any golden Lorentzian manifold equipped with generalized symmetric metric $U$-connection.

\begin{tabular}{lc}
\hline Connection type & Ricci tensor \\
\hline$\alpha$ semi-symmetric & $S\left(Y_{2}, Y_{3}\right)-\alpha^{2}(n \varepsilon-1) g\left(Y_{2}, Y_{3}\right)$ \\
\hline$\beta$ quarter symmetric & $S\left(Y_{2}, Y_{3}\right)+\beta^{2}\left\{g\left(\varphi Y_{2}, \varphi Y_{3}\right)-g\left(\varphi Y_{2}, Y_{3}\right)\right.$ trace $\left.\varphi\right\}$ \\
\hline Semi-symmetric & $S\left(Y_{2}, Y_{3}\right)-(n \varepsilon-1) g\left(Y_{2}, Y_{3}\right)$ \\
\hline Quarter symmetric & $S\left(Y_{2}, Y_{3}\right)+\left\{g\left(\varphi Y_{2}, \varphi Y_{3}\right)-g\left(\varphi Y_{2}, Y_{3}\right)\right.$ trace $\left.\varphi\right\}$ \\
\hline Connection type & Scalar curvature \\
\hline$\alpha$ semi-symmetric & $\tau-\alpha^{2}(n-\varepsilon) n$ \\
\hline$\beta$ quarter symmetric & $\tau+\beta^{2}\left\{\operatorname{trace} \varphi+n \varepsilon-(\operatorname{trace} \varphi)^{2}\right\}$ \\
\hline Semi-symmetric & $\tau-n(n-\varepsilon)$ \\
\hline Quarter symmetric & $\tau+\left\{\operatorname{trace} \varphi+n \varepsilon-(\operatorname{trace} \varphi)^{2}\right\}$ \\
\hline
\end{tabular}

Using Equations (3) and (17), we have

$$
\begin{aligned}
\bar{\tau}= & \frac{(\mp \sqrt{5}+3) c_{1}+( \pm \sqrt{5}+3) c_{2}-10 \alpha^{2}}{10}(n-\varepsilon) n \\
& +\frac{( \pm \sqrt{5}-1) c_{1}+(\mp \sqrt{5}-1) c_{2}-10 \alpha \beta}{10}[(2 n \varepsilon-2) \operatorname{trace} \varphi] \\
& +\frac{c_{1}+c_{2}-5 \beta^{2}}{5}\left[(\operatorname{trace} \varphi)^{2}-\operatorname{trace} \varphi-n \varepsilon\right] .
\end{aligned}
$$

Using Equation (23) in Theorem 3, we have the following theorem.

Theorem 4. For any locally golden product Lorentzian manifold equipped with generalized symmetric metric U-connection, we have the following characterizations in Table 2.

Table 2. Characterizations of any locally golden product Lorentzian manifold equipped with generalized symmetric metric $U$-connection.

\begin{tabular}{lc}
\hline Connection type & Scalar curvature \\
\hline$\alpha$ semi-symmetric & $\left(A-\alpha^{2}\right)(n-\varepsilon) n+B(2 n \varepsilon-2) \operatorname{trace} \varphi+C\left((\operatorname{trace} \varphi)^{2}-\operatorname{trace} \varphi-n \varepsilon\right)$ \\
\hline$\beta$ quarter symmetric & $A(n-\varepsilon) n+B(2 n \varepsilon-2) \operatorname{trace} \varphi+\left(C-\beta^{2}\right)\left((\operatorname{trace} \varphi)^{2}-\operatorname{trace} \varphi-n \varepsilon\right)$ \\
\hline Semi-symmetric & $(A-1)(n-\varepsilon) n+B(2 n \varepsilon-2) \operatorname{trace} \varphi+C\left((\operatorname{trace} \varphi)^{2}-\operatorname{trace} \varphi-n \varepsilon\right)$ \\
\hline Quarter symmetric & $A(n-\varepsilon) n+B(2 n \varepsilon-2) \operatorname{trace} \varphi+(C-1)\left((\operatorname{trace} \varphi)^{2}-\operatorname{trace} \varphi-n \varepsilon\right)$ \\
\hline where $A=\frac{(\mp \sqrt{5}+3) c_{1}+( \pm \sqrt{5}+3) c_{2}}{10}, B=\frac{( \pm \sqrt{5}-1) c_{1}+(\mp \sqrt{5}-1) c_{2}}{10}$ and $C=\frac{c_{1}+c_{2}}{5}$.
\end{tabular}




\section{Inequalities for Golden Lorentzian Manifolds Equipped with Generalized Symmetric Metric $U$-Connection}

From now on, let $M^{n}$ stand for locally golden product Lorentzian manifold with g.s.m. $U$-connection.

Theorem 5. For submanifold $N^{m}$ of $M^{n}$, we have the following inequalities

(i) for $\delta_{c}(r ; m-1)$,

$$
\begin{aligned}
\rho \leq & \frac{1}{\left(m^{2}-m\right)}\left[\delta_{c}(r ; m-1)\right] \\
& +\frac{(\mp \sqrt{5}+3) c_{1}+( \pm \sqrt{5}+3) c_{2}-10 \alpha^{2}}{10(m-1)}(m-\varepsilon) \\
& +\frac{( \pm \sqrt{5}-1) c_{1}+(\mp \sqrt{5}-1) c_{2}-10 \alpha \beta}{10\left(m^{2}-m\right)}[(2 m \varepsilon-2) \text { trace } \varphi] \\
& +\frac{c_{1}+c_{2}-5 \beta^{2}}{5\left(m^{2}-m\right)}\left[(\operatorname{trace} \varphi)^{2}-\operatorname{trace} \varphi-m \varepsilon\right]
\end{aligned}
$$

where the real number $r$ satisfies $\left(m^{2}-m\right)>r>0$;

(ii) for $\widehat{\delta}_{c}(r ; m-1)$,

$$
\begin{aligned}
\rho \leq & \frac{1}{\left(m^{2}-m\right)}\left[\widehat{\delta}_{c}(r ; m-1)\right] \\
& +\frac{(\mp \sqrt{5}+3) c_{1}+( \pm \sqrt{5}+3) c_{2}-10 \alpha^{2}}{10(m-1)}(m-\varepsilon) \\
& +\frac{( \pm \sqrt{5}-1) c_{1}+(\mp \sqrt{5}-1) c_{2}-10 \alpha \beta}{10\left(m^{2}-m\right)}[(2 m \varepsilon-2) \operatorname{trace} \varphi] \\
& +\frac{c_{1}+c_{2}-5 \beta^{2}}{5\left(m^{2}-m\right)}\left[(\operatorname{trace} \varphi)^{2}-\operatorname{trace} \varphi-m \varepsilon\right],
\end{aligned}
$$

where $\left(m^{2}-m\right)<r$.

In addition, the relations in Equations (18) and (19) become equalities if in an orthonormal frame $\left\{E_{1}, \ldots\right.$,

$\left.E_{m}, E_{m+1}, \ldots, E_{n}\right\}$, the shape operator can be represented as follows:

$$
S_{m+1}=\left(\begin{array}{cccccc}
b & 0 & 0 & \ldots & 0 & 0 \\
0 & b & 0 & \ldots & 0 & 0 \\
0 & 0 & b & \ldots & 0 & 0 \\
\vdots & \vdots & \vdots & \ddots & \vdots & \vdots \\
0 & 0 & 0 & \ldots & b & 0 \\
0 & 0 & 0 & \ldots & 0 & \frac{b m}{r}(m-1)
\end{array}\right), S_{m+2}=\cdots=S_{n}=0 .
$$

Proof. (i) For the locally golden product Lorentzian manifold $M$ equipped with g.s.m. $U$-connection, considering the orthonormal frame $\left\{E_{1}, \ldots, E_{m}, E_{m+1}, \ldots, E_{n}\right\}$ and using the Gauss equation and the relations in Equations (1) and (17), one achieves 


$$
\begin{aligned}
2 \bar{\tau}(p)= & \frac{(\mp \sqrt{5}+3) c_{1}+( \pm \sqrt{5}+3) c_{2}-10 \alpha^{2}}{10}(n-\varepsilon) n \\
& +\frac{( \pm \sqrt{5}-1) c_{1}+(\mp \sqrt{5}-1) c_{2}-10 \alpha \beta}{10}[(2 n \varepsilon-2) \text { trace } \varphi] \\
& +\frac{c_{1}+c_{2}-5 \beta^{2}}{5}\left[(\text { trace } \varphi)^{2}-\operatorname{trace} \varphi-n \varepsilon\right]+n^{2}\|\mathcal{H}\|^{2}-n \mathcal{C} .
\end{aligned}
$$

We write a quadratic polynomial $\mathcal{T}$ as follows

$$
\begin{aligned}
\mathcal{T}= & r \mathcal{C}+\frac{(n-1)\left(n^{2}-n-r\right)(n+r)}{r n} \mathcal{C}(\mathcal{J})-2 \bar{\tau}(p) \\
& +\frac{(\mp \sqrt{5}+3) c_{1}+( \pm \sqrt{5}+3) c_{2}-10 \alpha^{2}}{10}(n-\varepsilon) n \\
& +\frac{( \pm \sqrt{5}-1) c_{1}+(\mp \sqrt{5}-1) c_{2}-10 \alpha \beta}{10}[(2 n \varepsilon-2) \text { trace } \varphi] \\
& +\frac{c_{1}+c_{2}-5 \beta^{2}}{5}\left[(\operatorname{trace} \varphi)^{2}-\operatorname{trace} \varphi-n \varepsilon\right]
\end{aligned}
$$

where $\mathcal{J}$ stands for a hyperplane of $T_{p} N$. Now, consider the situation where, without loss of generality, $\left\{E_{1}, \ldots, E_{m-1}\right\}$ spans $\mathcal{J}$. In this way, we get

$$
\begin{aligned}
\mathcal{T}= & \frac{r}{m} \sum_{\alpha=m+1}^{n} \sum_{i, j=1}^{m}\left(h_{i j}^{\alpha}\right)^{2}+\left(\frac{1}{m}+\frac{1}{r}\right)\left(m^{2}-m-r\right) \sum_{\alpha=m+1}^{n} \sum_{i, j=1}^{m-1}\left(h_{i j}^{\alpha}\right)^{2} \\
& -2 \bar{\tau}(p)+\frac{(\mp \sqrt{5}+3) c_{1}+( \pm \sqrt{5}+3) c_{2}-10 \alpha^{2}}{10}(n-\varepsilon) n \\
& +\frac{( \pm \sqrt{5}-1) c_{1}+(\mp \sqrt{5}-1) c_{2}-10 \alpha \beta}{10}[(2 n \varepsilon-2) \text { trace } \varphi] \\
& +\frac{c_{1}+c_{2}-5 \beta^{2}}{5}\left[(\operatorname{trace} \varphi)^{2}-\operatorname{trace} \varphi-n \varepsilon\right] .
\end{aligned}
$$

It follows that

$$
\begin{aligned}
\mathcal{T}= & \left(\frac{r}{m}+1\right) \sum_{\alpha=m+1}^{n} \sum_{i, j=1}^{m}\left(h_{i j}^{\alpha}\right)^{2}+\left(\frac{1}{m}+\frac{1}{r}\right)\left(m^{2}-m-r\right) \sum_{\alpha=m+1}^{n} \sum_{i, j=1}^{m-1}\left(h_{i j}^{\alpha}\right)^{2} \\
& -\sum_{\alpha=m+1}^{n}\left(\sum_{i=1}^{m} h_{i i}^{\alpha}\right)^{2} .
\end{aligned}
$$

This leads us to the following

$$
\begin{aligned}
\mathcal{T}= & \sum_{\alpha=m+1}^{n} \sum_{i=1}^{m-1}\left[\left\{\frac{m^{2}}{r}+\frac{(r-1)}{r} m-2\right\}\left(h_{i i}^{\alpha}\right)^{2}+\frac{2 r+2 m}{m}\left(h_{i m}^{\alpha}\right)^{2}\right] \\
& +\sum_{\alpha=m+1}^{n}\left[\frac{2 r+2 m}{r}(m-1) \sum_{i<j=1}^{m-1}\left(h_{i j}^{\alpha}\right)^{2}-2 \sum_{i<j=1}^{m} h_{i i}^{\alpha} h_{j j}^{\alpha}\right. \\
& \left.+\frac{r}{m}\left(h_{m m}^{\alpha}\right)^{2}\right] .
\end{aligned}
$$

With the help of Equation (21), the critical points

$$
h^{c}=\left(h_{11}^{m+1}, h_{12}^{m+1}, \ldots, h_{m m}^{m+1}, \ldots, h_{11}^{n}, \ldots, h_{m m}^{n}\right)
$$


are the solutions of (for $1 \leq i, j \leq m-1$ )

$$
\begin{aligned}
\frac{\partial \mathcal{T}}{\partial h_{i i}^{\alpha}} & =2\left[\frac{(r+m)}{r}(m-1) h_{i i}^{\alpha}-\sum_{l=1}^{m} h_{l l}^{\alpha}\right]=0, \\
\frac{\partial \mathcal{T}}{\partial h_{m m}^{\alpha}} & =\frac{2 r}{m} h_{m m}^{\alpha}-2 \sum_{l=1}^{m-1} h_{l l}^{\alpha}=0, \\
\frac{\partial \mathcal{T}}{\partial h_{i j}^{\alpha}} & =\frac{(m+r)}{r}(4 m-4) h_{i j}^{\alpha}=0, \\
\frac{\partial \mathcal{T}}{\partial h_{i m}^{\alpha}} & =\frac{4 r+4 m}{m} h_{i m}^{\alpha}=0,
\end{aligned}
$$

$\forall \alpha \in\{m+1, \ldots, n\}$ and $i \neq j$.

When $i$ is not equal to $j$, Equation (22) produces $h_{i j}^{\alpha}=0$ for every solution of $h^{c}$, and the first two sets of equations in Equation (22) have the determinant equal to zero (submanifolds that are not totally geodesic have solutions). Apart from this, one can express the Hessian matrix by

$$
H(\mathcal{T})=\left(\begin{array}{ccc}
H_{1} & 0 & 0 \\
0 & H_{2} & 0 \\
0 & 0 & H_{3}
\end{array}\right)
$$

where

$$
H_{1}=\left(\begin{array}{ccccc}
\frac{2(r+m)(m-1)}{r}-2 & -2 & \cdots & -2 & -2 \\
-2 & \frac{2(m-1)(r+m)}{r}-2 & \ldots & -2 & -2 \\
\vdots & \vdots & \ddots & \vdots & \vdots \\
-2 & -2 & \ldots & \frac{2(r+m)(m-1)}{r}-2 & -2 \\
-2 & -2 & \ldots & -2 & \frac{2 r}{m}
\end{array}\right),
$$

and 0 stands for the null matrices of corresponding sizes. We also write

$$
\begin{gathered}
H_{2}=\operatorname{diag}\left(\frac{2}{r}(r+m)(m-1), \frac{2}{r}(m-1)(r+m), \ldots, \frac{2}{r}(r+m)(m-1)\right), \\
H_{3}=\operatorname{diag}\left(\frac{2(r+m)}{m}, \frac{2(r+m)}{m}, \ldots, \frac{2(r+m)}{m}\right) .
\end{gathered}
$$

In this way, we find the following eigenvalues for $H(\mathcal{T})$

$$
\begin{gathered}
\lambda_{11}=0, \lambda_{22}=\frac{2\left(m^{3}-m^{2}+r^{2}\right)}{r m}, \lambda_{33}=\cdots=\lambda_{m m}=\frac{2(m-1)(r+m)}{r}, \\
\lambda_{i j}=\frac{2}{r}(m-1)(r+m), \lambda_{i n}=\frac{2(r+m)}{m},
\end{gathered}
$$

where in we have assumed that $i \neq j$.

This concludes $\mathcal{T}$ to be parabolic and confirms its approach to a minimum $\mathcal{T}\left(h^{c}\right)$ at any solution $h^{\mathcal{c}}$ of Equation (22). Now, Equations (21) and (22) lead us to $\mathcal{T}\left(h^{\mathcal{c}}\right)=0$, establishing $\mathcal{T} \geq 0$ and providing the following 


$$
\begin{aligned}
2 \bar{\tau}(p) \leq & r \mathcal{C}+\frac{(m-1)\left(m^{2}-m-r\right)(m+r)}{r m} \mathcal{C}(\mathcal{J}) \\
& +\frac{(\mp \sqrt{5}+3) c_{1}+( \pm \sqrt{5}+3) c_{2}-10 \alpha^{2}}{10}(m-\varepsilon) m \\
& +\frac{( \pm \sqrt{5}-1) c_{1}+(\mp \sqrt{5}-1) c_{2}-10 \alpha \beta}{10}[(2 m \varepsilon-2) \text { trace } \varphi] \\
& +\frac{c_{1}+c_{2}-5 \beta^{2}}{5}\left[(\operatorname{trace} \varphi)^{2}-\operatorname{trace} \varphi-m \varepsilon\right]
\end{aligned}
$$

where by, we get

$$
\begin{aligned}
\rho \leq & \mathcal{C} \frac{r}{\left(m^{2}-m\right)}+\frac{(r+m)}{r m^{2}}\left(m^{2}-m-r\right) \mathcal{C}(\mathcal{J}) \\
& +\frac{(\mp \sqrt{5}+3) c_{1}+( \pm \sqrt{5}+3) c_{2}-10 \alpha^{2}}{10(m-1)}(m-\varepsilon) \\
& +\frac{( \pm \sqrt{5}-1) c_{1}+(\mp \sqrt{5}-1) c_{2}-10 \alpha \beta}{10\left(m^{2}-m\right)}[(2 m \varepsilon-2) \text { trace } \varphi] \\
& +\frac{c_{1}+c_{2}-5 \beta^{2}}{5\left(m^{2}-m\right)}\left[(\operatorname{trace} \varphi)^{2}-\operatorname{trace} \varphi-m \varepsilon\right]
\end{aligned}
$$

where $\mathcal{J}$ stands for any tangent hyperplane of $T_{p} N$ such that Equation (18) holds in view of the above equation. In addition, $\forall i, j \in\{1, \ldots m\}$ with $i \neq j$, Equation (18) holds for equality if

$$
h_{i j}^{\alpha}=0,
$$

and

$$
h_{m m}^{\alpha}=\frac{(m-1)}{r} m h_{11}^{\alpha}=\frac{(m-1)}{r} m h_{22}^{\alpha} \cdots=\frac{(m-1)}{r} m h_{m-1 m-1}^{\alpha},
$$

Therefore, by virtue of Equations (23) and (24), one concludes that with trivial normal connection, $N$ is invariantly quasi-umbilical in $M^{n}$ if and only if Equation (18) holds for equality, in such a way that $S$ appears as Equation (20) for some local orthonormal frames.

(ii) Equation (19) can also be established in a similar fashion.

Next, we write the following result.

Corollary 2. For any submanifold $N^{m}$ immersed in a locally golden product Lorentzian manifold $M^{n}$ endowed with a generalized symmetric metric U-connection, the following inequalities hold

(i) for $\delta_{c}(m-1)$;

$$
\begin{aligned}
\rho \leq & \delta_{c}(m-1) \\
& +\frac{(\mp \sqrt{5}+3) c_{1}+( \pm \sqrt{5}+3) c_{2}-10 \alpha^{2}}{10(m-1)}(m-\varepsilon) \\
& +\frac{( \pm \sqrt{5}-1) c_{1}+(\mp \sqrt{5}-1) c_{2}-10 \alpha \beta}{10\left(m^{2}-m\right)}[(2 m \varepsilon-2) \text { trace } \varphi] \\
& +\frac{c_{1}+c_{2}-5 \beta^{2}}{5\left(m^{2}-m\right)}\left[(\operatorname{trace} \varphi)^{2}-\operatorname{trace} \varphi-m \varepsilon\right],
\end{aligned}
$$

where $0 \leq r \leq\left(m^{2}-m\right)$; 
(ii) for $\widehat{\delta}_{\mathcal{c}}(m-1)$;

$$
\begin{aligned}
\rho \leq & \widehat{\delta}_{c}(m-1) \\
& +\frac{(\mp \sqrt{5}+3) c_{1}+( \pm \sqrt{5}+3) c_{2}-10 \alpha^{2}}{10(m-1)}(m-\varepsilon) \\
& +\frac{( \pm \sqrt{5}-1) c_{1}+(\mp \sqrt{5}-1) c_{2}-10 \alpha \beta}{10\left(m^{2}-m\right)}[(2 m \varepsilon-2) \text { trace } \varphi] \\
& +\frac{c_{1}+c_{2}-5 \beta^{2}}{5\left(m^{2}-m\right)}\left[(\operatorname{trace} \varphi)^{2}-\operatorname{trace} \varphi-m \varepsilon\right],
\end{aligned}
$$

where $\left(m^{2}-m\right)<r$.

In addition, Equations (25) and (26) hold for equality if for an orthonormal frame $\left\{E_{1}, \ldots\right.$, $\left.E_{m}, E_{m+1}, \ldots, E_{n}\right\}$, operator $S$ can be represented as follows

$$
S_{m+1}=\left(\begin{array}{cccccc}
b & 0 & 0 & \ldots & 0 & 0 \\
0 & b & 0 & \ldots & 0 & 0 \\
0 & 0 & b & \ldots & 0 & 0 \\
\vdots & \vdots & \vdots & \ddots & \vdots & \vdots \\
0 & 0 & 0 & \ldots & b & 0 \\
0 & 0 & 0 & \ldots & 0 & 2 b
\end{array}\right), \quad S_{m+2}=\ldots=S_{n}=0 ;
$$

and

$$
S_{m+1}=\left(\begin{array}{cccccc}
2 b & 0 & 0 & \ldots & 0 & 0 \\
0 & 2 b & 0 & \ldots & 0 & 0 \\
0 & 0 & 2 b & \ldots & 0 & 0 \\
\vdots & \vdots & \vdots & \ddots & \vdots & \vdots \\
0 & 0 & 0 & \ldots & 2 b & 0 \\
0 & 0 & 0 & \ldots & 0 & b
\end{array}\right), \quad S_{m+2}=\ldots=S_{n}=0
$$

Now, we will give some results for the theorem 5

\section{Some Consequences}

Corollary 3. For a Riemannian manifold $N^{m}$ isometrically immersed in $M^{n}$, we have the following:

(i) for $\delta_{c}(r ; m-1)$, for every $r \in\{0, \ldots, m(m-1)\}$ :

(a) $\quad M^{n}$ is equipped with a semi-symmetric metric U-connection

$$
\begin{aligned}
\rho \leq & \frac{1}{\left(m^{2}-m\right)}\left[\delta_{\mathcal{c}}(r ; m-1)\right] \\
& +\frac{(\mp \sqrt{5}+3) c_{1}+( \pm \sqrt{5}+3) c_{2}-10 \alpha^{2}}{10(m-1)}(m-\varepsilon) \\
& +\frac{( \pm \sqrt{5}-1) c_{1}+(\mp \sqrt{5}-1) c_{2}}{10\left(m^{2}-m\right)}[(2 m \varepsilon-2) \text { trace } \varphi] \\
& +\frac{c_{1}+c_{2}}{5\left(m^{2}-m\right)}\left[(\operatorname{trace} \varphi)^{2}-\operatorname{trace} \varphi-m \varepsilon\right],
\end{aligned}
$$


(b) $\quad M^{n}$ is equipped with $\beta$ quarter symmetric metric U-connection

$$
\begin{aligned}
\rho \leq & \frac{1}{\left(m^{2}-m\right)}\left[\delta_{c}(r ; m-1)\right] \\
& +\frac{(\mp \sqrt{5}+3) c_{1}+( \pm \sqrt{5}+3) c_{2}}{10(m-1)}(m-\varepsilon) \\
& +\frac{( \pm \sqrt{5}-1) c_{1}+(\mp \sqrt{5}-1) c_{2}}{10\left(m^{2}-m\right)}[(2 m \varepsilon-2) \operatorname{trace} \varphi] \\
& +\frac{c_{1}+c_{2}-5 \beta^{2}}{5\left(m^{2}-m\right)}\left[(\operatorname{trace} \varphi)^{2}-\operatorname{trace} \varphi-m \varepsilon\right]
\end{aligned}
$$

(c) $\quad M^{n}$ is equipped with semi-symmetric metric U-connection

$$
\begin{aligned}
\rho \leq & \frac{1}{\left(m^{2}-m\right)}\left[\delta_{c}(r ; m-1)\right] \\
& +\frac{(\mp \sqrt{5}+3) c_{1}+( \pm \sqrt{5}+3) c_{2}-10}{10(m-1)}(m-\varepsilon) \\
& +\frac{( \pm \sqrt{5}-1) c_{1}+(\mp \sqrt{5}-1) c_{2}}{10\left(m^{2}-m\right)}[(2 m \varepsilon-2) \operatorname{trace} \varphi] \\
& +\frac{c_{1}+c_{2}}{5\left(m^{2}-m\right)}\left[(\operatorname{trace} \varphi)^{2}-\operatorname{trace} \varphi-m \varepsilon\right]
\end{aligned}
$$

(d) $\quad M^{n}$ is equipped with quarter symmetric metric U-connection

$$
\begin{aligned}
\rho \leq & \frac{1}{\left(m^{2}-m\right)}\left[\delta_{c}(r ; m-1)\right] \\
& +\frac{(\mp \sqrt{5}+3) c_{1}+( \pm \sqrt{5}+3) c_{2}}{10(m-1)}(m-\varepsilon) \\
& +\frac{( \pm \sqrt{5}-1) c_{1}+(\mp \sqrt{5}-1) c_{2}}{10\left(m^{2}-m\right)}[(2 m \varepsilon-2) \text { trace } \varphi] \\
& +\frac{c_{1}+c_{2}-5}{5\left(m^{2}-m\right)}\left[(\operatorname{trace} \varphi)^{2}-\operatorname{trace} \varphi-m \varepsilon\right] .
\end{aligned}
$$

(ii) for $\widehat{\delta}_{c}(r ; m-1)$, for every $r>m(m-1)$ :

(a) $\quad M^{n}$ is equipped with a semi-symmetric metric U-connection

$$
\begin{aligned}
\rho \leq & \frac{1}{\left(m^{2}-m\right)}\left[\widehat{\delta}_{c}(r ; m-1)\right] \\
& +\frac{(\mp \sqrt{5}+3) c_{1}+( \pm \sqrt{5}+3) c_{2}-10 \alpha^{2}}{10(m-1)}(m-\varepsilon) \\
& +\frac{( \pm \sqrt{5}-1) c_{1}+(\mp \sqrt{5}-1) c_{2}}{10\left(m^{2}-m\right)}[(2 m \varepsilon-2) \operatorname{trace} \varphi] \\
& +\frac{c_{1}+c_{2}}{5\left(m^{2}-m\right)}\left[(\operatorname{trace} \varphi)^{2}-\operatorname{trace} \varphi-m \varepsilon\right]
\end{aligned}
$$


(b) $\quad M^{n}$ is equipped with $\beta$ quarter symmetric metric U-connection

$$
\begin{aligned}
\rho \leq & \frac{1}{\left(m^{2}-m\right)}\left[\widehat{\delta}_{c}(r ; m-1)\right] \\
& +\frac{(\mp \sqrt{5}+3) c_{1}+( \pm \sqrt{5}+3) c_{2}}{10(m-1)}(m-\varepsilon) \\
& +\frac{( \pm \sqrt{5}-1) c_{1}+(\mp \sqrt{5}-1) c_{2}}{10\left(m^{2}-m\right)}[(2 m \varepsilon-2) \operatorname{trace} \varphi] \\
& +\frac{c_{1}+c_{2}-5 \beta^{2}}{5\left(m^{2}-m\right)}\left[(\operatorname{trace} \varphi)^{2}-\operatorname{trace} \varphi-m \varepsilon\right]
\end{aligned}
$$

(c) $\quad M^{n}$ is equipped with semi-symmetric metric U-connection

$$
\begin{aligned}
\rho \leq & \frac{1}{\left(m^{2}-m\right)}\left[\widehat{\delta}_{c}(r ; m-1)\right] \\
& +\frac{(\mp \sqrt{5}+3) c_{1}+( \pm \sqrt{5}+3) c_{2}-10}{10(m-1)}(m-\varepsilon) \\
& +\frac{( \pm \sqrt{5}-1) c_{1}+(\mp \sqrt{5}-1) c_{2}}{10\left(m^{2}-m\right)}[(2 m \varepsilon-2) \operatorname{trace} \varphi] \\
& +\frac{c_{1}+c_{2}}{5\left(m^{2}-m\right)}\left[(\operatorname{trace} \varphi)^{2}-\operatorname{trace} \varphi-m \varepsilon\right]
\end{aligned}
$$

(d) $\quad M^{n}$ is equipped with quarter symmetric metric U-connection

$$
\begin{aligned}
\rho \leq & \frac{1}{\left(m^{2}-m\right)}\left[\widehat{\delta}_{c}(r ; m-1)\right] \\
& +\frac{(\mp \sqrt{5}+3) c_{1}+( \pm \sqrt{5}+3) c_{2}}{10(m-1)}(m-\varepsilon) \\
& +\frac{( \pm \sqrt{5}-1) c_{1}+(\mp \sqrt{5}-1) c_{2}}{10\left(m^{2}-m\right)}[(2 m \varepsilon-2) \text { trace } \varphi] \\
& +\frac{c_{1}+c_{2}-5}{5\left(m^{2}-m\right)}\left[(\operatorname{trace} \varphi)^{2}-\operatorname{trace} \varphi-m \varepsilon\right] .
\end{aligned}
$$

Moreover, the relations in the above results become equalities if in some orthonormal frame $\left\{E_{1}, \ldots, E_{m}, E_{m+1}, \ldots, E_{n}\right\}$, the operator $S$ reduces to:

$$
S_{m+1}=\left(\begin{array}{cccccc}
b & 0 & 0 & \ldots & 0 & 0 \\
0 & b & 0 & \ldots & 0 & 0 \\
0 & 0 & b & \ldots & 0 & 0 \\
\vdots & \vdots & \vdots & \ddots & \vdots & \vdots \\
0 & 0 & 0 & \ldots & b & 0 \\
0 & 0 & 0 & \ldots & 0 & \frac{b\left(m^{2}-m\right)}{r}
\end{array}\right), \quad S_{m+2}=\cdots=S_{n}=0
$$

Corollary 4. When $N^{m}$ represents a Riemannian manifold isometrically immersed in a golden Lorentzian manifold $M^{n}$ equipped with a g.s.m. U-connection, we have the following relations (i) for $\delta_{c}(m-1)$, for every $r \in\left\{0, \ldots\left(m^{2}-m\right)\right\}$ : 
(a) $\quad M^{n}$ is equipped with $\alpha$ semi-symmetric metric $U$-connection

$$
\begin{aligned}
\rho \leq & \frac{1}{\left(m^{2}-m\right)}\left[\delta_{c}(m-1)\right] \\
& +\frac{(\mp \sqrt{5}+3) c_{1}+( \pm \sqrt{5}+3) c_{2}-10 \alpha^{2}}{10(m-1)}(m-\varepsilon) \\
& +\frac{( \pm \sqrt{5}-1) c_{1}+(\mp \sqrt{5}-1) c_{2}}{10\left(m^{2}-m\right)}[(2 m \varepsilon-2) \text { trace } \varphi] \\
& +\frac{c_{1}+c_{2}}{5\left(m^{2}-m\right)}\left[(\operatorname{trace} \varphi)^{2}-\operatorname{trace} \varphi-m \varepsilon\right]
\end{aligned}
$$

(b) $\quad M^{n}$ is equipped with $\beta$ quarter symmetric metric $U$-connection

$$
\begin{aligned}
\rho \leq & \frac{1}{\left(m^{2}-m\right)}\left[\delta_{c}(m-1)\right] \\
& +\frac{(\mp \sqrt{5}+3) c_{1}+( \pm \sqrt{5}+3) c_{2}}{10(m-1)}(m-\varepsilon) \\
& +\frac{( \pm \sqrt{5}-1) c_{1}+(\mp \sqrt{5}-1) c_{2}}{10\left(m^{2}-m\right)}[(2 m \varepsilon-2) \operatorname{trace} \varphi] \\
& +\frac{c_{1}+c_{2}-5 \beta^{2}}{5\left(m^{2}-m\right)}\left[(\operatorname{trace} \varphi)^{2}-\operatorname{trace} \varphi-m \varepsilon\right]
\end{aligned}
$$

(c) $\quad M^{n}$ is equipped with semi-symmetric metric U-connection

$$
\begin{aligned}
\rho \leq & \frac{1}{\left(m^{2}-m\right)}\left[\delta_{c}(m-1)\right] \\
& +\frac{(\mp \sqrt{5}+3) c_{1}+( \pm \sqrt{5}+3) c_{2}-10}{10(m-1)}(m-\varepsilon) \\
& +\frac{( \pm \sqrt{5}-1) c_{1}+(\mp \sqrt{5}-1) c_{2}}{10\left(m^{2}-m\right)}[(2 m \varepsilon-2) \operatorname{trace} \varphi] \\
& +\frac{c_{1}+c_{2}}{5\left(m^{2}-m\right)}\left[(\operatorname{trace} \varphi)^{2}-\operatorname{trace} \varphi-m \varepsilon\right]
\end{aligned}
$$

(d) $\quad M^{n}$ is equipped with quarter symmetric metric U-connection

$$
\begin{aligned}
\rho \leq & \frac{1}{\left(m^{2}-m\right)}\left[\delta_{c}(m-1)\right] \\
& +\frac{(\mp \sqrt{5}+3) c_{1}+( \pm \sqrt{5}+3) c_{2}}{10(m-1)}(m-\varepsilon) \\
& +\frac{( \pm \sqrt{5}-1) c_{1}+(\mp \sqrt{5}-1) c_{2}}{10\left(m^{2}-m\right)}[(2 m \varepsilon-2) \text { trace } \varphi] \\
& +\frac{c_{1}+c_{2}-5}{5\left(m^{2}-m\right)}\left[(\operatorname{trace} \varphi)^{2}-\operatorname{trace} \varphi-m \varepsilon\right] .
\end{aligned}
$$

(ii) for $\widehat{\delta}_{c}(m-1)$, for every $\left(m^{2}-m\right)<r$. 
(a) $\quad M^{n}$ is equipped with $\alpha$ semi-symmetric metric $U$-connection

$$
\begin{aligned}
\rho \leq & \frac{1}{\left(m^{2}-m\right)}\left[\widehat{\delta}_{\mathcal{c}}(m-1)\right] \\
& +\frac{(\mp \sqrt{5}+3) c_{1}+( \pm \sqrt{5}+3) c_{2}-10 \alpha^{2}}{10(m-1)}(m-\varepsilon) \\
& +\frac{( \pm \sqrt{5}-1) c_{1}+(\mp \sqrt{5}-1) c_{2}}{10\left(m^{2}-m\right)}[(2 m \varepsilon-2) \operatorname{trace} \varphi] \\
& +\frac{c_{1}+c_{2}}{5\left(m^{2}-m\right)}\left[(\operatorname{trace} \varphi)^{2}-\operatorname{trace} \varphi-m \varepsilon\right]
\end{aligned}
$$

(b) $\quad M^{n}$ is equipped with $\beta$ quarter symmetric metric $U$-connection

$$
\begin{aligned}
\rho \leq & \frac{1}{\left(m^{2}-m\right)}\left[\widehat{\delta}_{c}(m-1)\right] \\
& +\frac{(\mp \sqrt{5}+3) c_{1}+( \pm \sqrt{5}+3) c_{2}}{10(m-1)}(m-\varepsilon) \\
& +\frac{( \pm \sqrt{5}-1) c_{1}+(\mp \sqrt{5}-1) c_{2}}{10\left(m^{2}-m\right)}[(2 m \varepsilon-2) \operatorname{trace} \varphi] \\
& +\frac{c_{1}+c_{2}-5 \beta^{2}}{5\left(m^{2}-m\right)}\left[(\operatorname{trace} \varphi)^{2}-\operatorname{trace} \varphi-m \varepsilon\right]
\end{aligned}
$$

(c) $\quad M^{n}$ is equipped with semi-symmetric metric U-connection

$$
\begin{aligned}
\rho \leq & \frac{1}{\left(m^{2}-m\right)}\left[\widehat{\delta}_{c}(m-1)\right] \\
& +\frac{(\mp \sqrt{5}+3) c_{1}+( \pm \sqrt{5}+3) c_{2}-10}{10(m-1)}(m-\varepsilon) \\
& +\frac{( \pm \sqrt{5}-1) c_{1}+(\mp \sqrt{5}-1) c_{2}}{10\left(m^{2}-m\right)}[(2 m \varepsilon-2) \operatorname{trace} \varphi] \\
& +\frac{c_{1}+c_{2}}{5\left(m^{2}-m\right)}\left[(\operatorname{trace} \varphi)^{2}-\operatorname{trace} \varphi-m \varepsilon\right]
\end{aligned}
$$

(d) $\quad M^{n}$ is equipped with quarter symmetric metric U-connection

$$
\begin{aligned}
\rho \leq & \frac{1}{\left(m^{2}-m\right)}\left[\widehat{\delta}_{c}(m-1)\right] \\
& ++\frac{(\mp \sqrt{5}+3) c_{1}+( \pm \sqrt{5}+3) c_{2}}{10(m-1)}(m-\varepsilon) \\
& +\frac{( \pm \sqrt{5}-1) c_{1}+(\mp \sqrt{5}-1) c_{2}}{10\left(m^{2}-m\right)}[(2 m \varepsilon-2) \text { trace } \varphi] \\
& +\frac{c_{1}+c_{2}-5}{5\left(m^{2}-m\right)}\left[(\operatorname{trace} \varphi)^{2}-\operatorname{trace} \varphi-m \varepsilon\right] .
\end{aligned}
$$


Equalities hold for all relations in the above results if in some orthonormal frame $\left\{E_{1}, \ldots, E_{m}\right.$, $\left.E_{m+1}, \ldots, E_{n}\right\}$, the shape operators take the following form

$$
S_{m+1}=\left(\begin{array}{cccccc}
b & 0 & 0 & \ldots & 0 & 0 \\
0 & b & 0 & \ldots & 0 & 0 \\
0 & 0 & b & \ldots & 0 & 0 \\
\vdots & \vdots & \vdots & \ddots & \vdots & \vdots \\
0 & 0 & 0 & \ldots & b & 0 \\
0 & 0 & 0 & \ldots & 0 & 2 b
\end{array}\right), \quad S_{m+2}=\cdots=S_{n}=0
$$

and

$$
S_{m+1}=\left(\begin{array}{cccccc}
2 b & 0 & 0 & \ldots & 0 & 0 \\
0 & 2 b & 0 & \ldots & 0 & 0 \\
0 & 0 & 2 b & \ldots & 0 & 0 \\
\vdots & \vdots & \vdots & \ddots & \vdots & \vdots \\
0 & 0 & 0 & \ldots & 2 b & 0 \\
0 & 0 & 0 & \ldots & 0 & b
\end{array}\right), \quad S_{m+2}=\cdots=S_{n}=0
$$

Remark 1. The proofs of all the corollaries are similar to that of Theorem 5.

Remark 2. For a Lorentzian manifold M equipped with generalized symmetric metric U-connection, we can define structures of different types [4]:

- $\quad$ silver type $\sigma_{2,1}=1+\sqrt{2}$ if $p=2$ and $q=1$;

- subtle type $\sigma_{4,1}=2+\sqrt{5}$ if $p=4$ and $q=1$;

- copper type $\sigma_{1,2}=2$ with $p=1$ and $q=2$;

- $\quad$ bronze type $\sigma_{3,1}=\frac{3+\sqrt{13}}{2}$ with $p=3$ and $q=1$;

- $\quad$ nickel type $\sigma_{1,3}=\frac{1+\sqrt{13}}{2}$ if $p=1$ and $q=3$, etc.

Using the above structures, one can establish inequalities similar to Theorem 5.

\section{Discussion}

The present study deals with generalized symmetric metric $U$-connection on golden Lorentzian manifolds. We also study lower bounds for submanifolds immersed in golden Lorentzian manifolds equipped with generalized symmetric metric $U$-connection. Moreover, submanifolds for which equality holds are also discussed.

Author Contributions: Data curation, M.A.C. and M.D.S.; Funding acquisition, K.M.K.; Investigation, M.A.C. and O.B.; Project administration, O.B.; Software, O.B.; Writing — review and editing, M.D.S. All authors have read and agreed to the published version of the manuscript.

Funding: The second author received funding through the research group program under grant number R.G.P.1/50/42 from the deanship of Scientific research at King Khalid University, KSA.

Institutional Review Board Statement: Not applicable.

Informed Consent Statement: Not applicable.

Data Availability Statement: Not applicable.

Acknowledgments: The authors thank the reviewers for their valuable and constructive comments for modifying the presentation of this work. The authors extend their appreciation to the deanship of Scientific research at King Khalid University for funding through the research group program under grant number R.G.P.1/50/42.

Conflicts of Interest: The authors declare no conflict of interest. 
Sample Availability: Not Applicable.

\section{References}

1. Crasmareanu, M.; Hretcanu, C. Golden differential geometry. Chaos Solitons Fractals 2008, 38, 1229-1238. [CrossRef]

2. Goldberg, S.I.; Petridids, N.C. Differentiable solutions of algebraic equations on manifolds. Kodai Math. Semin. Rep. 1973, 25, 111-128. [CrossRef]

3. Goldberg, S.I.; Yano, K. Polynomial structures on manifolds. Kodai Math. Semin. Rep. 1970, 22, 199-218. [CrossRef]

4. Hretcanu, C.; Crasmareanu, M. On some invariant submanifolds in a Riemannian manifold with golden structure. An. Stiins. Univ. Al. I. Cuza Iasi. Mat. (N.S.) 2007, 53, 199-211.

5. Ahmad, M.; Qayyoom, M.A. On submanifolds in a Riemannian manifold with golden structure. Turk. J. Math. Comput. Sci. 2019, 11, 8-23.

6. Bahadir, O.; Uddin, S. Slant submanifolds of Golden Riemannian manifolds. J. Math. Ext. 2019, 13, 1-10.

7. Ozkan, M. Prolongations of golden structures to tangent bundles. In Differential Geometry Dynamical Systems; World Scientific: Singapore, 2014; Volume 16, pp. 227-238.

8. Gezer, A.; Cengiz, N.; Salimov, A. On integrability of Golden Riemannian structures. Turk. J. Math. 2013, 37, 693-703.

9. Hretcanu, C.; Crasmareanu, M. Applications of the golden ratio on Riemannian manifolds. Turk. J. Math. 2009, 33, 179-191.

10. Hretcanu, C. Submanifolds in Riemannian manifold with Golden structure. In Proceedings of the Workshop on Finsler Geometry and Its Applications, Balatonfured, Hungary, 28 May-2 June 2007.

11. Choudhary, M.A.; Blaga, A.M. Generalized Wintgen inequality for slant submanifolds in metallic Riemannian space forms. J. Geom. 2021, 112, 26. [CrossRef]

12. Chen, B.Y. Some pinching and classification theorems for minimal submanifolds. Arch. Math. 1993, 60, 568-578. [CrossRef]

13. Choudhary, M.A.; Park, K. Optimization on slant submanifolds of golden Riemannian manifolds using generalized normalized $\delta$-Casorati curvatures. J. Geom. 2020, 111, 31. [CrossRef]

14. Mihai, I.; Al-Solamy F.R.; Shahid, M.H. On Ricci curvature of a quaternion CR-submanifold in a quaternion space form. Rad. Mat. 2003, 12, 91-98.

15. Shukla, S.S.; Rao, P.K. Ricci curvature of quaternion slant submanifolds in quaternion space forms. Acta Math. Acad. Paedagog. Nyhzi 2012, 28, 69-81.

16. Casorati, F. Mesure de la courbure des surfaces suivant l'idée commune. Acta Math. 1890, 14, 95-110. [CrossRef]

17. Decu, S.; Haesen, S.; Verstraelen, L. Optimal inequalities involving Casorati curvatures. Bull. Transilv. Univ. Braşov Ser. III Math. Inform. Phys. 2007, 14, 85-93.

18. Ghisoiu, V. Inequalities for the Casorati curvatures of slant submanifolds in complex space forms. In Proceedings of the Conference RIGA Riemannian Geometry and Applications, Bucharest, Romania, 10-14 May 2011.

19. Decu, S.; Haesen, S.; Verstraelen, L. Optimal inequalities characterising quasi-umbilical submanifolds. J. Inequal. Pure Appl. Math. 2008, 9, 79 .

20. Lee, C.W.; Vilcu G.E. Optimal inequalities for the normalized $\delta$-Casorati curvatures of submanifolds in Kenmotsu space forms. Adv. Geom. 2017, 17, 1-13. [CrossRef]

21. Lee, C.W.; Lee, J.W.; Vilcu, G.E.; Yoon, D.W. Optimal inequalities for the Casorati curvatures of the submanifolds of generalized space form endowed with semi-symmetric metric connections. Bull. Korean Math. Soc. 2015, 52, 1631-1647. [CrossRef]

22. Choudhary, M.A.; Blaga A.M. Inequalities for generalized normalized $\delta$-Casorati curvatures of slant submanifolds in metallic Riemannian space forms. J. Geom. 2020, 111, 1-18. [CrossRef]

23. Chen, B.Y. Pseudo-Riemannian Geometry, S-Invariants and Applications; World Scientific: Hackensack, NJ, USA, 2011.

24. Blair, D.E. Quasi-umbilical, minimal submanifolds of Euclidean space. Simon Stevin 1977, 51, 3-22. 